\title{
Large-Eddy Simulation of Turbulent Flow over Wavy Wall with Different Wave Steepness
}

\author{
Enwei Zhang, Xiaoliang Wang and Qingquan Liu* \\ Department of Mechanics, School of Aerospace Engineering, Beijing Institute of Technology, Beijing \\ 100081, China
}

\begin{abstract}
Large-eddy simulation (LES) with dynamic one-equation subgrid-scale stress model was utilized to study the characteristics of turbulent flow over sea-surface generalized as two-dimensional wavy wall with different wave steepness. The statistical characteristics of turbulent field and pressure distribution were presented in detail. The simulation results showed that the separation zones induce stronger convection as the wave steepness increases. Reynolds shear stress near the wall boundary showed positive correlation with the wave steepness, while in the outer region, time-averaged turbulent fields seemed to be insensitive with the wall boundary. The equivalent velocity profiles obtained from the spatial averaging of the time-averaged velocity indicated that lower velocity occurred as the increase of wave steepness due to the increased pressureinduced form drag. The vortex structures were also visualized and showed vertically-bent characteristics which induced negative Reynolds shear stress at the stoss side as the increase of wave steepness that is equivalent to the effect of wave age.
\end{abstract}

\section{Introduction}

A large variety of dynamic processes in nature such as atmospheric flow over topography, current flow over seabed or airflow over water waves happen naturally (Fernando, 2010). Especially for airflow over sea surface with water waves, the momentum transport become complex due to the impact of complex sea wave. Many studies have been conducted about the flow characteristics over complex geometry boundary by experimental study or numerical simulation. A frequent-used method to simplify the wall geometry is to regard the boundary as two-dimensional wavy wall (Buckles, Hanratty, and Adrian, 1984). Consequently, a large variety of experiments could be conducted to obtain the turbulent fields (Hudson, Dykhno, and Hanratty, 1996, Nakagawa, and Hanratty, 2001, Günther, and von Rohr, 2003). Although these studies gave detailed description of the flow characteristics, however, how the vortex structures impact the turbulent fields with different wave steepness (or the ratio of amplitude to wavelength) was not discussed in detail.

Therefore, Michioka (2018) numerically studied the turbulent flow and gas dispersion over wavy wall with different ratio of amplitude to wavelength, and found the large standard deviations of the fluctuation of streamwise and spanwise velocity appearing

\footnotetext{
* Corresponding author: liuqq@bit.edu.cn
} 
obviously at the windward of the wavy wall related to the Görtler vortices. Different from the flat wall boundary layer, Hamed et al. (2015), by experiments, found that negative Reynolds shear stress occurred at stoss side when flowing over wavy wall boundary and it is the reason by using cartesian coordinate system (Hudson, Dykhno, and Hanratty, 1996, Cherukat, Hanratty, and Mclaughlin, 1998). While another explanation by Yang and Shen (2009) was that quasi-streamwise vortices at stoss side with ejection events induces negative Reynolds shear stress. However, these studies considered just one parameter of wave steepness and lacked of a mechanism about how wave steepness impact the flow characteristics and the vortex structures.

This study presents a large-eddy simulation of turbulent flow over wavy wall with different wave steepness. The statistical characteristics of turbulent field, the time-averaged and spatial-averaged turbulent fields and pressure distribution were discussed in detail. And the vortex structures were extracted to explain the impact on high-order statistics of turbulent fields.

\section{Method}

In this study, the large-eddy simulation with dynamic one-equation subgrid-scale stress model was used. The filtered incompressible N-S equations and the continuity equation for describing resolvable turbulent flow field are as follow:

$$
\begin{aligned}
& \frac{\partial u_{i}}{\partial x_{i}}=0 \\
& \frac{\partial u_{i}}{\partial t}+u_{j} \frac{\partial u_{i}}{\partial x_{j}}=-\frac{1}{\rho} \frac{\partial p}{\partial x_{i}}+v \nabla^{2} u_{i}-\frac{\partial \tau_{i j}}{\partial x_{j}}
\end{aligned}
$$

$x_{i}(i=1,2,3)$ represent $x, y, z$ directions corresponding to streamwise, spanwise and vertical directions, $u_{i}(u, v, w)$ are the filtered velocity components, $p$ is the filtered pressure, $v$ represents kinematic viscosity and $\tau_{i j}$ is subgrid-scale stress tensor modelled by dynamic one-equation model (Kim, and Menon, 1995) that expresses as:

$$
\begin{aligned}
& \tau_{i j}=-\frac{L_{i j} M_{i j}}{2 M_{i j} M_{i j}} \Delta K^{1 / 2}\left(\frac{\partial u_{i}}{\partial x_{j}}+\frac{\partial u_{j}}{\partial x_{i}}\right)+\frac{2}{3} \delta_{i j} K \\
& L_{i j}=\widetilde{u_{i} u_{j}}-\widetilde{u_{i}} \widetilde{u_{j}}, \\
& M_{i j}=-\frac{\widetilde{\Delta}}{2}\left[\frac{1}{2}\left(\widetilde{u_{i} u_{j}}-\widetilde{u_{i}} \widetilde{u_{j}}\right)\right]^{1 / 2}\left(\frac{\partial \widetilde{u_{i}}}{\partial x_{j}}+\frac{\partial \widetilde{u_{j}}}{\partial x_{i}}\right) \\
& \frac{\partial K}{\partial t}+u_{i} \frac{\partial K}{\partial x_{i}}=-\tau_{i j} \frac{\partial u_{i}}{\partial x_{j}}-v\left(\frac{\partial u_{i}}{\partial x_{j}} \frac{\partial u_{i}}{\partial x_{j}}-\frac{\widetilde{\partial u_{i}}}{\partial x_{j}} \frac{\partial u_{i}}{\partial x_{j}}\right)\left(\frac{1}{2} L_{i i}\right)^{-3 / 2} \frac{\widetilde{\Delta}}{\Delta} K^{3 / 2}+\frac{\partial}{\partial x_{i}}\left(\frac{1}{2} \frac{L_{i j} M_{i j}}{M_{i j} M_{i j}} \Delta K^{1 / 2} \frac{\partial K}{\partial x_{i}}\right),
\end{aligned}
$$


where, the $\Delta=\sqrt[3]{\Delta x \Delta y \Delta z}$ is the filtered grid scale. $K$ represents subgrid-scale kinetic energy which can be resolved by the transport equation (6). The : represents test filter that $\stackrel{\complement}{\Delta}=2 \Delta$, and the $\mathfrak{l}^{\mathrm{c}}$ is the filtered velocity in test filter. The OpenFOAM is used for numerical simulation with PimpleFoam solver that SIMPLE algorithm is carried for pressure-velocity coupling. The numerical verification can be found in detail in our previous work (Zhang et al. 2021).

\section{Physical model and flow parameter}

In present study, the wall boundary was generalized as sinusoidal wave with four different wave steepness defined as $a k(=0.157,0.314,0.471,0.628)$. Here, $a$ is the amplitude and $k$ is the wave number. The flow above the boundary wall is driven by external force with periodic boundary conditions along $x, y$ directions. No-slip wall is applied at top and bottom boundaries. The computational domain is $(x / \lambda, y / \lambda, z / h)=(2,0.5,1)$ that is large enough to capture flow structures by two-points spatial correlation. The Reynolds number is defined as $\operatorname{Re}=U_{\text {in }} h^{\prime} / v$, the $h^{\prime}$ is the half height of the domain and $U_{\text {in }}$ is bulk velocity. The first layer of grids is set as $\Delta z^{+}=0.5<1$ to make sure enough accuracy of boundary layer. After the turbulent flow fully develops, time-averaging is carried out for another additional thirty flow periods.

\section{Results}

Figure 1 illustrates the contour of time-averaged turbulent fields. The distribution of streamwise velocity near the wall show wavy-spatial distribution as the increase of wave steepness. In the separation zones, the vertical velocity shows different direction at trough with downwelling motion at lee side, while upwelling motion at stoss side (behind the reattachment point). As shown in figure 1(c1)-(c4), the turbulent kinetic energy shows high correlation with the $a k$, and the region of higher turbulent level corresponds to the separation zones, revealing the relation between turbulent level with strong convection. In addition, the Reynolds shear stress shows similar characteristics with turbulent kinetic energy as illustrated in figure 1(d1)-(d4), but it should be noted that the Reynolds shear stress at stoss side near the wall is negative that is related with the quasi-streamwise vortices (Yang, and Shen, 2009). 

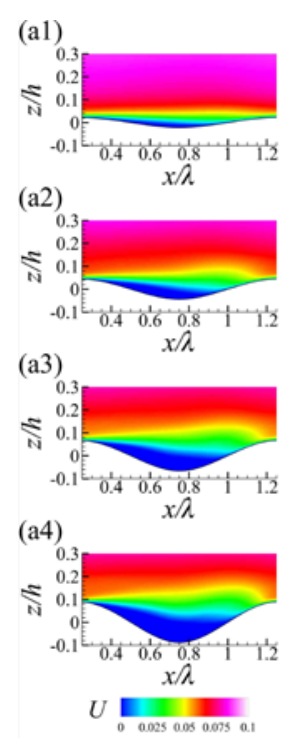

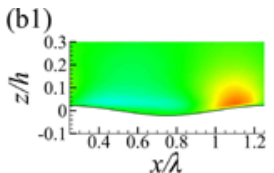

(b2)

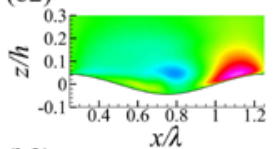

(b3)
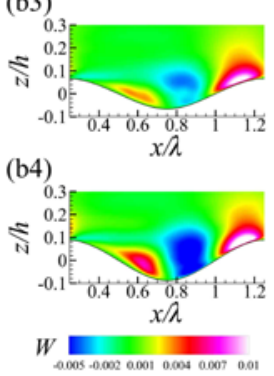

(c1)

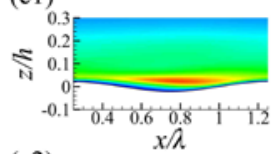

(c2)

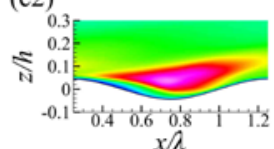

(c3)

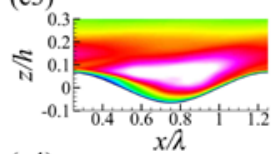

(c4)

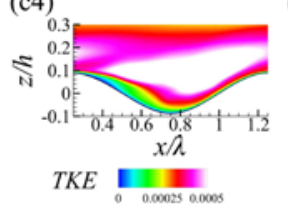

(d1)

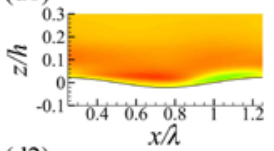

(d2)

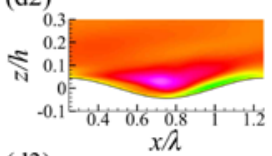

(d3)

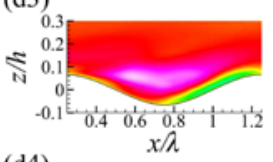

(d4)

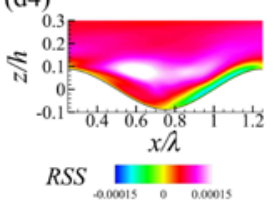

Fig. 1. Contour of time-averaged turbulent field, series (a) represents time-averaged streamwise velocity, the series (b) are time-averaged vertical velocity, series (c) and (d) are turbulent kinetic energy and Reynolds shear stress, respectively, the mark 1-4 represent different wave steepness with $a k(=0.157,0.314,0.471,0.628)$.

Figure 2 clearly shows the separation zones with streamlines patterned like closedellipse. The separation regions locate at $x / \lambda:[0.3,0.55],[0.2,0.7],[0.15,0.75],[0.1,0.8]$ for increasing $a k$, therefore, increased wave steepness leads to upstream shift of the separation point and downstream shift of the reattachment point. To quantitively examine the impact of wave steepness on turbulent kinetic energy, the profiles at different $x$ positions ((a) to (d) represent profiles at different $x$ position with $x / \lambda=0.2,0.4,0.6,0.8)$ are given in figure 3 . The increased turbulent kinetic energy occurs as the increase of wave steepness which is consistent with results of figure 1(c1)-(c4), besides, the maximum of turbulent kinetic energy locate at $z / h=0.1$ for $a k=0.628$ at $x / \lambda=0.2$ as illustrated in figure 3(a), with downward shift as the wave steepness decreases at same $x$ position.

Figure 4 quantitively gives the Reynolds shear stress profiles for different wave steepness at $x / \lambda=0: 0.9$. As the results show, the profiles in the outer region at $z / h>0.2$ collapse well. Due to the non-flatness of the boundary geometry, the average wall shear stress cannot be obtained directly. So, the method proposed by Hudson, Dykhno, and Hanratty (1996) is carried out by extrapolating Reynolds shear stress from outer layer to inner region at $z / h=0$ as the blue dashed lines shown in figure 4 . Then the friction velocity $u_{\tau}$ can be obtained by equation (7).

$$
u_{\tau}=\left(\frac{\tau_{\text {wall }}}{\rho}\right)^{1 / 2}=\left(\frac{-\left.\rho\left\langle u^{\prime} w^{\prime}\right\rangle\right|_{z=0}}{\rho}\right)^{1 / 2}=\left(-\left.\left\langle u^{\prime} w^{\prime}\right\rangle\right|_{z=0}\right)^{1 / 2}
$$




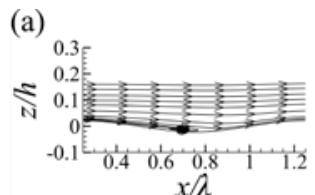

(c)
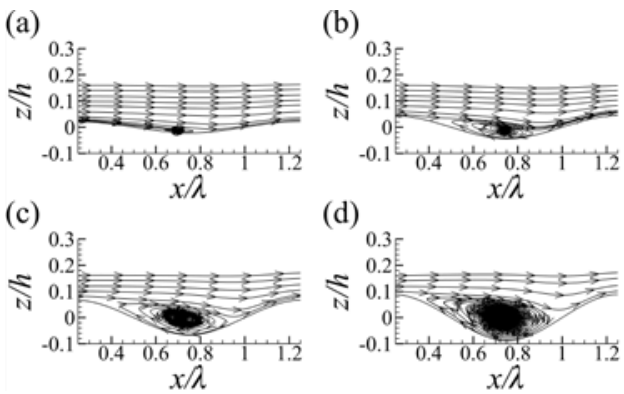

(d)

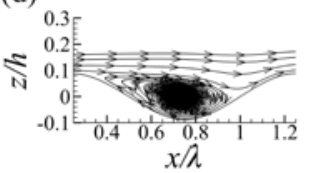

Fig. 2. Time-averaged streamlines for different cases.

(a)

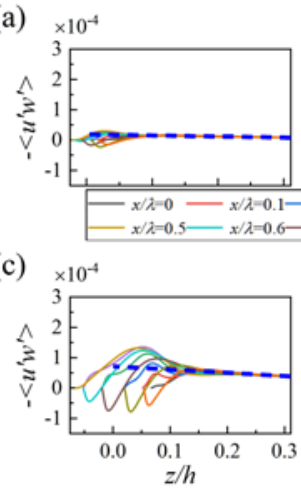

(b) $\times 10^{-4}$

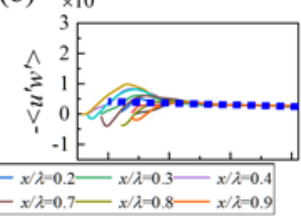

(d)

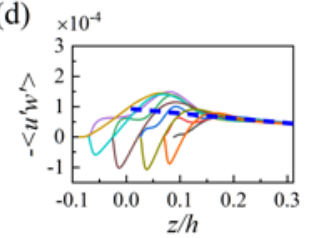

Fig. 4. Reynolds shear stress profiles along one wavelength for different cases.
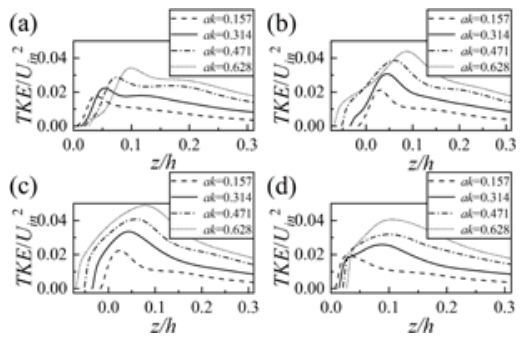

Fig. 3. TKE profiles along one wavelength for different cases.

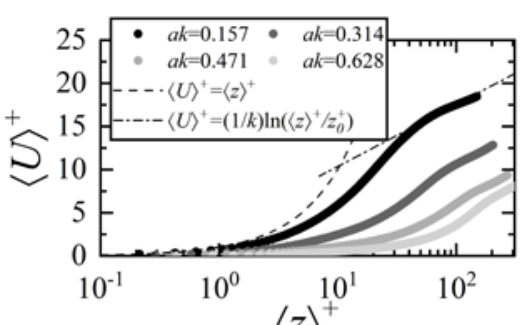

$\langle z\rangle$

Fig. 5. Spatial and time averaging of streamwise velocity along one wavelength for different cases, the linear plot of flat wall boundary layer is also given as the dash line shows.

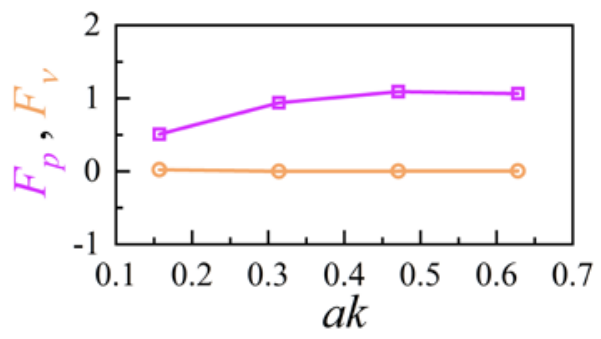

Fig. 7. Pressure-induced form drag and viscous drag vary as a function of wave steepness. (a)

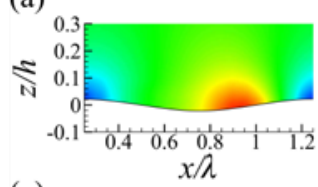

(c)

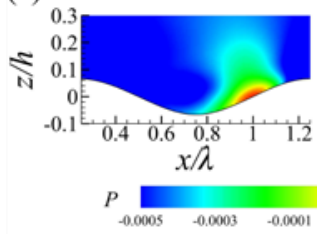

(d)
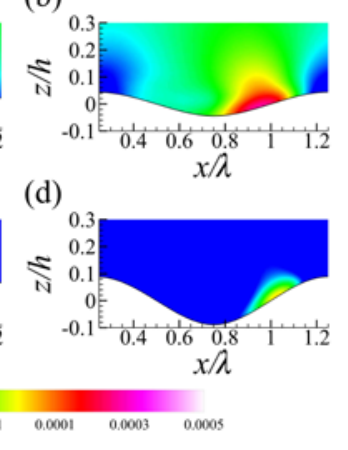

Fig. 6. Time-averaged pressure distribution for different cases.

(b)

(d) (1)

In order to compare the velocity profile of wavy wall with flat wall boundary layer, spatial averaging of time-averaged velocity was implemented along one wavelength as figure 5 shows. But attention should be paid that the spatial averaging of velocity is an equivalent variable instead of real quantity. The dashed line represents linear law in viscous 
sublayer for flat wall boundary layer at $\langle z\rangle^{+}<5$ (Kim, Moin, and Moser, 1987), and the dot dashed line is the logarithmic law in logarithmic region (+ represents dimensionless by $u_{\tau}$ for velocity scale and $v / u_{\tau}$ for length scale). As the increase of wave steepness, logarithmic region extends into the range of $\langle z\rangle^{+}=50: 150$, besides, profile of velocity decreases with down shift compared with flat wall boundary layer in both viscous sublayer and logarithmic region.

In addition, the pressure distribution for different wave steepness is given in figure 6 . Reduced positive pressure region can be observed at stoss side as the increase of wave steepness, while the enhanced negative pressure region locates at lee side. The dimensionless pressure-induced form drag and viscous drag then can be obtained by $F_{\mathrm{p}}=\int_{0}^{\lambda} \frac{P}{\rho u_{\tau}^{2} \lambda} \frac{\partial z}{\partial x} \mathrm{~d} x$ and $F_{v}=\int_{0}^{\lambda} \frac{\tau_{\mathrm{v}}}{\rho u_{\tau}^{2} \lambda} \frac{\partial z}{\partial x} \mathrm{~d} x$. As figure 7 shows, the pressure-induced form drag increases as the increase of wave steepness, while the magnitude of viscous drag can be ignored. The form drag at wall boundary reduces the flow velocity in viscous sublayer, while the different profile of velocity in logarithmic region is owing to the recirculation bubble in separation zones.

(a)

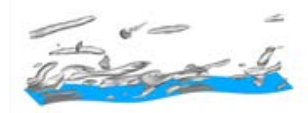

(c)

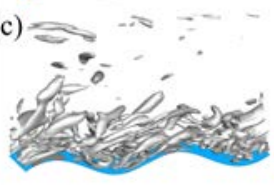

Fig. 8. Instantaneous vortex structures visualized by $Q$ criterion with $Q=5$ for different cases. (d)

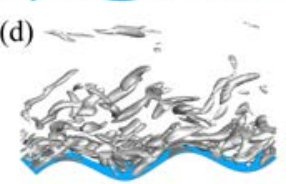

(b)

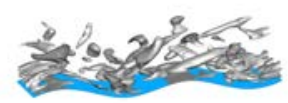

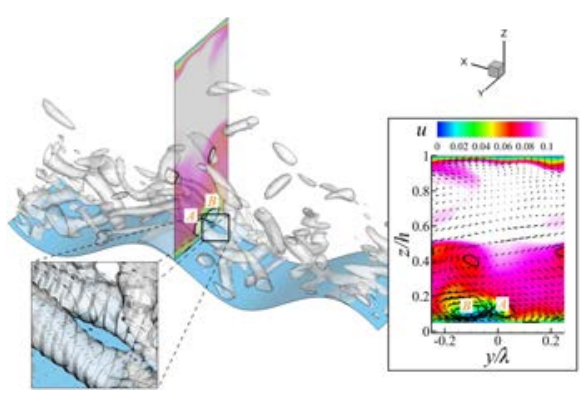

Fig. 9. Vortex pair at stoss side with $Q=10$ for case $a k=0.628$, and the vector in $3 \mathrm{D}$ view is $(u, v, w)$. Section locates at $x / \lambda=1$ with vector $(v, w)$ shown. The vortex pair is depicted by $\mathrm{A}$ and $\mathrm{B}$ that the vorticity is $\omega_{\mathrm{Ax}}<0$ and $\omega_{\mathrm{B} x}>0$.

The turbulent field is known to be largely affected by the large-scale vortex structures. To clearly understand the impact of vortex on the turbulent field, the vortex structures are extracted by $Q$ criterion in the instantaneous velocity field. And the $Q$ is defined as the residual of the vorticity tensor norm squared subtracted from the strain-rate tensor norm squared that can be expressed as:

$$
Q=\frac{1}{2}\left(\|\boldsymbol{\Omega}\|_{\mathrm{F}}^{2}-\|\mathbf{S}\|_{\mathrm{F}}^{2}\right)
$$

where, the $\mathbf{S}=0.5\left(\nabla \mathbf{u}+\nabla \mathbf{u}^{\mathrm{T}}\right)$ is strain-rate tensor and $\mathbf{\Omega}=0.5\left(\nabla \mathbf{u}-\nabla \mathbf{u}^{\mathrm{T}}\right)$ is vorticity tensor. The vortex occurs at a region with $Q>0$. Figure 8 shows the vortex structures of instantaneous turbulent field with $Q=5$. The quasi-streamwise vortices dominate under the condition of lower wave steepness, while vertically-bent vortices occur especially at trough for higher wave steepness as shown in figure $8(\mathrm{~d})$. Where vertically-bent vortex 
striding across the crest can be clearly observed at stoss side as the increase of wave steepness. To discuss how the vortex structure impact the turbulent field at stoss side for large wave steepness case, figure 9 shows the vortex of $Q=10$ and a section located at $x / \lambda: 1$ with instantaneous streamwise velocity and vector $(v, w)$ to depict the vorticity near the wall boundary at stoss side. The vortex pair A and B show counter-rotating feature with $\omega_{\mathrm{A} x}<0$ and $\omega_{\mathrm{B} x}>0$ (the vorticity can be calculated by $\omega=\frac{\partial u_{i}}{\partial x_{j}}-\frac{\partial u_{j}}{\partial x_{i}}$ ), inducing the ejection event along $+z$ direction, consequently, stimulating the enhanced negative Reynolds shear stress.

\section{Discussion}

The simulation results indicate that wave steepness impacts significantly on the flow characteristics that stronger convection occurs with high wave steepness due to flow separation. Besides, the enhanced turbulent level with higher turbulent kinetic energy and Reynolds shear stress also shows high correlation with the wave steepness. The increased wave steepness results in higher pressure-induced form drag, giving rise to reduced spatialaveraged velocity with profile shifting downward. By visualizing instantaneous vortex structures, quasi-streamwise vortex gradually bents vertically as the increase of wave steepness which is equivalent to the impact of wave age.

\section{Conclusions}

The study presents a numerical simulation to analyse the impact of wave steepness on turbulent flow. In conclusion, the wave steepness highly impact turbulence flowing over wavy wall boundary, especially at trough with strong convection due to flow separation and at stoss side with enhanced shear layer due to the vertically-bent vortex structures. Therefore, the impact of wave steepness that is as equivalent as the wave age's effect should be more considered.

\section{Acknowledgments}

The authors are grateful for the support of the National Natural Science Foundation of China (No. 12032005).

\section{References}

[1] Fernando H J S 2010 Fluid Dynamics of Urban Atmospheres in Complex Terrain Annu. Rev. Fluid Mech. 42 365-389

[2] Buckles J, Hanratty T J and Adrian R J 1984 Turbulent flow over large amplitude wavy surfaces J. Fluid Mech. 140 27-44

[3] Hudson J D, Dykhno L and Hanratty T J 1996 Turbulence production in flow over a wavy wall Exp. Fluids 20 257-265

[4] Nakagawa S and Hanratty T J 2001 Particle image velocimetry measurements of flow over a wavy wall Phys. Fluids 13(11) 3504-3507 
[5] Günther A and von Rohr P R 2003 Large-scale structures in a developed flow over a wavy wall $J$. Fluid Mech. 478 257-285

[6] Michioka T 2018 Large-eddy simulation for turbulent flow and gas dispersion over wavy walls Int. J. Heat Mass Tran. 125 569-579

[7] Hamed A H, Kamdar A, Castillo L and Chamorro L P 2015 Turbulent boundary layer over 2D and 3D large-scale wavy walls Phys. Fluids 27(10) 106601

[8] Cherukat P, Hanratty T J and Mclaughlin J B 1998 Direct numerical simulation of a fully developed turbulent flow over a wavy wall Theor. Comp. Fluid Dyn. 11 109134

[9] Yang D and Shen L 2009 Characteristics of coherent vortical structures in turbulent flows over progressive surface waves Phys. Fluids 21125106

[10] Kim W and Menon S 1995 A New Dynamic One-Equation Subgrid Scale Model for Large Eddy Simulations in: 33rd Aerospace Sciences Meeting and Exhibit, American Institute of Aeronautics and Astronautics, Reno, NV, USA, 1-9

[11] Zhang E W, Wang X L and Liu Q Q 2021 Effects of the spanwise heterogeneity of a three-dimensional wavy wall on momentum and scalar transport Phys. Fluids 33 055116

[12] Kim J, Moin P and Moser R 1987 Turbulence statistics in fully developed channel flow at low Reynolds number J. Fluid Mech. 177 133-166 